Homology, Homotopy and Applications, vol.10(2), 2008, pp.175-193

\title{
ADDING INVERSES TO DIAGRAMS II: INVERTIBLE HOMOTOPY THEORIES ARE SPACES
}

\author{
JULIA E. BERGNER
}

\author{
(communicated by J. F. Jardine)
}

\begin{abstract}
In previous work, we showed that there are appropriate model category structures on the category of simplicial categories and on the category of Segal precategories, and that they are Quillen equivalent to one another and to Rezk's complete Segal space model structure on the category of simplicial spaces. Here, we show that these results still hold if we instead use groupoid or "invertible" cases. Namely, we show that model structures on the categories of simplicial groupoids, Segal pregroupoids, and invertible simplicial spaces are all Quillen equivalent to one another and to the standard model structure on the category of spaces. We prove this result using two different approaches to invertible complete Segal spaces and Segal groupoids.
\end{abstract}

\section{Introduction}

The notion of homotopy theories as mathematical objects is becoming a useful tool in topology as more mathematical structures are being viewed from a homotopical or higher-categorical viewpoint. Currently, there are four known models for homotopy theories: simplicial categories, Segal categories, complete Segal spaces, and quasi-categories. There are corresponding model category structures for each; the first three were shown to be Quillen equivalent to each other by the author [7], and the fourth was shown to be equivalent to the first by Joyal [19]; explicit equivalences between the fourth and the other two are also given by Joyal and Tierney [21]. Each of these models has proved to be useful in different contexts. Simplicial categories are naturally models for homotopy theories, in that they arise naturally from model categories, or more generally from categories with weak equivalences, via Dwyer and Kan's simplicial localization techniques $[\mathbf{1 1}, \mathbf{1 3}]$. For this reason, one important motivation for studying any of these models is to understand specific homotopy theories and relationships between them. Quasi-categories, on the other hand, are more clearly a generalization of categories and more suited to constructions that look like those

Received October 11, 2007, revised June 10, 2008; published on October 22, 2008.

2000 Mathematics Subject Classification: 55U35, 18G30, 18 E35.

Key words and phrases: homotopy theories, simplicial categories, simplicial groupoids, complete Segal spaces, Segal groupoids, model categories, $(\infty, 1)$-categories and groupoids.

This article is available at http://intlpress.com/HHA/v10/n2/a9

Copyright (c) 2008, International Press. Permission to copy for private use granted. 
appearing in category theory. In fact, both Joyal [20] and Lurie [22] have written extensively on extending category theory to quasi-category theory. The objects in all four models are often called $(\infty, 1)$-categories, to indicate that they can be regarded as categories with $n$-morphisms for any $n \geqslant 1$, but for which these $n$-morphisms are all invertible whenever $n>1$.

In this current paper, we would like to show that the first three models can be restricted to the groupoid case without much difficulty. Such structures could be called $(\infty, 1)$-groupoids, but they are really $(\infty, 0)$-categories, since even the 1 -morphisms are invertible in this case. In fact, we go on to prove that these model structures are Quillen equivalent to the standard model structure on the category of simplicial sets (and therefore to the standard model structure on the category of topological spaces). It has been proposed by a number of people, beginning with Grothendieck [16], that $\infty$-groupoids, or $(\infty, 0)$-categories, should be models for homotopy types of spaces, so this result can be seen as further evidence for this "homotopy hypothesis." Many authors have proved results in this area, including Tamsamani [29], Berger [3], Cisinski [10], Paoli [24], Biedermann [8], and Barwick [2], and a nice overview is given by Baez $[\mathbf{1}]$.

We should further note here that this comparison actually encompasses an invertible version of the fourth model, that of quasi-categories, since a quasi-category with inverses is just a Kan complex, and the fibrant objects in the standard model category structure on the category of simplicial sets are precisely the Kan complexes.

\section{Organization}

In Section 2, we give a new proof of the existence of a model structure on the category of simplicial groupoids. In Sections 3 and 4, we define invertible versions of complete Segal spaces and Segal categories using the category $\mathbf{I} \boldsymbol{\Delta}^{o p}$ rather than $\boldsymbol{\Delta}^{o p}$ as a means of encoding inverses. We prove the existence of appropriate model category structures as well. In Section 5, we prove that these simplicial groupoid, Segal groupoid, and invertible complete Segal space model structures are Quillen equivalent to one another and to the standard model category structure on the category of simplicial sets. In Section 6, we give an alternate approach to invertible versions of Segal categories and complete Segal spaces by changing the projection maps in the category $\boldsymbol{\Delta}^{o p}$, and we again show that we have a zig-zag of Quillen equivalences between the resulting model categories.

We refer the reader to the previous paper [4] for our notations and conventions regarding simplicial objects and model categories.

\section{Acknowledgements}

Support from the Fields Institute for a visit in May 2007, when much of the work for this paper was completed, is gratefully acknowledged. I would like to thank André Joyal and Simona Paoli for discussions on the material in this paper, as well as the referee for suggestions for its improvement. 


\section{A model category structure on the category of simplicial groupoids}

A simplicial category is a category $\mathcal{C}$ enriched over simplicial sets, or a category such that, for objects $x$ and $y$ of $\mathcal{C}$, there is a simplicial set of morphisms $\operatorname{Map}_{\mathcal{C}}(x, y)$ between them.

Recall that the category of components $\pi_{0} \mathcal{C}$ of a simplicial category $\mathcal{C}$ is the category with the same objects as $\mathcal{C}$ and such that

$$
\operatorname{Hom}_{\pi_{0} \mathcal{C}}(x, y)=\pi_{0} \operatorname{Map}_{\mathcal{C}}(x, y) \text {. }
$$

We use the following notion of equivalence of simplicial categories.

Definition 2.1 ([11, 2.4]). A functor $f: \mathcal{C} \rightarrow \mathcal{D}$ between two simplicial categories is a Dwyer-Kan equivalence if it satisfies the following two conditions:

- (W1) for any objects $x$ and $y$ of $\mathcal{C}$, the induced map

$$
\operatorname{Map}_{\mathcal{C}}(x, y) \rightarrow \operatorname{Map}_{\mathcal{D}}(f x, f y)
$$

is a weak equivalence of simplicial sets, and

- (W2) the induced map of categories of components $\pi_{0} f: \pi_{0} \mathcal{C} \rightarrow \pi_{0} \mathcal{D}$ is an equivalence of categories.

Dwyer and Kan proved in $[\mathbf{1 2}, 2.5]$ that there is a model structure on the category $\mathcal{S G}$ pd of small simplicial groupoids with the Dwyer-Kan equivalences as weak equivalences. In fact, they went on to show that this model structure has the additional structure of a simplicial model category, and that it is Quillen equivalent to the usual model structure on the category $\mathcal{S} \mathcal{S}$ ets of simplicial sets. In this section, we give an alternate proof of the existence of this model structure, following the proof for the model category of small simplicial categories [5].

Consider the category $\mathcal{S G} p d_{\mathcal{O}}$ of simplicial groupoids with a fixed set $\mathcal{O}$ of objects. In particular, the morphisms in this category are required to be the identity map on this set of objects. There is a model category structure on $\mathcal{S G} p d_{\mathcal{O}}$ in which the weak equivalences are defined to be those simplicial functors $f: \mathcal{C} \rightarrow \mathcal{D}$ such that for any objects $x$ and $y$ of $\mathcal{C}$, the map

$$
\operatorname{Map}_{\mathcal{C}}(x, y) \rightarrow \operatorname{Map}_{\mathcal{D}}(f x, f y)
$$

is a weak equivalence of simplicial sets. The fibrations are defined analogously [4]. This model category can also be shown to have the additional structure a simplicial model category, just as Dwyer and Kan show for the analogous model category $\mathcal{S C}_{\mathcal{O}}$ of small simplicial categories with a fixed object set [13, 7.1].

Theorem 2.2. The category $\mathcal{S G}$ pd of small simplicial groupoids has a cofibrantly generated model category structure given by the following three classes of morphisms:

1. The weak equivalences are the Dwyer-Kan equivalences of simplicial groupoids.

2. The fibrations are the maps $f: \mathcal{C} \rightarrow \mathcal{D}$ such that

- (F1) for any objects $x$ and $y$ of $\mathcal{C}$, the map

$$
\operatorname{Map}_{\mathcal{C}}(x, y) \rightarrow \operatorname{Map}_{\mathcal{D}}(f x, f y)
$$

is a fibration of simplicial sets, and 
- (F2) for any object $x$ in $\mathcal{C}, z$ in $\mathcal{D}$, and morphism $g: f x \rightarrow z$ in $\mathcal{D}_{0}$, there is an object $y$ in $\mathcal{C}$ and morphism $d: x \rightarrow y$ in $\mathcal{C}_{0}$ such that $f d=g$.

3. The cofibrations are the maps with the left lifting property with respect to the maps which are both fibrations and weak equivalences.

To prove this theorem, we need to define candidates for our sets of generating cofibrations and generating acyclic cofibrations. To do so, we begin by defining the functor

$$
U_{G}: \mathcal{S S} \text { ets } \rightarrow \mathcal{S G} \text { pd }
$$

Let $\mathcal{S C}$ denote the category of small simplicial categories. The functor $U_{G}$ is the composite of the functor $U: \mathcal{S S e t s} \rightarrow \mathcal{S C}$ taking a simplicial set $X$ to the simplicial category with objects $x$ and $y, \operatorname{Hom}(x, y)=X$, and no other nonidentity morphisms, and the functor $L: \mathcal{S C} \rightarrow \mathcal{S G} p d$ left adjoint to the forgetful functor.

Using this functor, we define the set of generating cofibrations to consist of the maps

- (C1) $U_{G} \dot{\Delta}[n] \rightarrow U_{G} \Delta[n]$ for all $n \geqslant 1$, and

- $(\mathrm{C} 2) \emptyset \rightarrow\{x\}$.

Similarly, we define the set of generating acyclic cofibrations to consist of the maps

- (A1) $U_{G} V[n, k] \rightarrow U_{G} \Delta[n]$ for all $n \geqslant 1,0 \leqslant k \leqslant n$, and

- (A2) $\{x\} \rightarrow \mathcal{F}$, where $\mathcal{F}$ is the groupoid with two objects $x$ and $y$ and with all the mapping spaces given by $\Delta[0]$.

Notice, in particular, that the set of generating acyclic cofibrations is substantially smaller than the analogous set for the model structure on the category of simplicial categories.

Proposition 2.3. A map $f: \mathcal{C} \rightarrow \mathcal{D}$ of simplicial groupoids has the right lifting property with respect to the maps in (A1) and (A2) if and only if it satisfies (F1) and (F2).

Proof. Using the standard model structure $\mathcal{S} \mathcal{S}$ ets on the category of simplicial sets, it is not hard to show that condition (F1) is equivalent to having the right lifting property with respect to the maps in (A1).

Thus, let us suppose that $f$ has the right lifting property with respect to (A1) and (A2) and show that $f$ satisfies (F2). In other words, given an object $x$ of $\mathcal{C}$ and object $z$ of $\mathcal{D}$, we need to show that a map $g: f x \rightarrow z$ in $\mathcal{D}$ lifts to a map $d: x \rightarrow y$ for some object $y$ of $\mathcal{C}$ such that $f y=z$ and $f d=g$. Let us consider the objects $w=f x$ and $z$ in $\mathcal{C}$.

First suppose that $w \neq z$. Define $\mathcal{E}$ to be the full simplicial subcategory of $\mathcal{D}$ with objects $w$ and $z$. Let $\mathcal{F}$ be the simplicial category with two objects $w$ and $z$ and a single isomorphism $h: w \rightarrow z$. Consider some map $i: \mathcal{F} \rightarrow \mathcal{E}$ which preserves the objects. Note that the map $\{x\} \rightarrow \mathcal{F}$ is precisely the map in (A2). Consider the 
composite inclusion map $\mathcal{F} \rightarrow \mathcal{E} \rightarrow \mathcal{D}$. These maps fit into a diagram

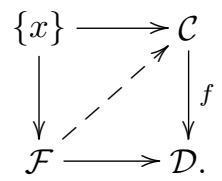

The dotted arrow lift exists because we assume that $f: \mathcal{C} \rightarrow \mathcal{D}$ has the right lifting property with respect to the map in (A2). But, the existence of this lift implies that any morphism in $\mathcal{D}$, since it must be an isomorphism, lifts to a morphism in $\mathcal{C}$.

Then, suppose that $w=z$. Define $\mathcal{E}^{\prime}$ to be the simplicial groupoid with two objects $w$ and $w^{\prime}$ such that each function complex of $\mathcal{E}^{\prime}$ is the $\operatorname{simplicial}$ set $\operatorname{Map}_{\mathcal{D}}(w, w)$ and composites are defined as they are in $\mathcal{D}$. We then define the map $\mathcal{E}^{\prime} \rightarrow \mathcal{D}$ which sends both objects of $\mathcal{E}^{\prime}$ to $w$ in $\mathcal{D}$ and is the identity map on all the function complexes. Using this simplicial groupoid $\mathcal{E}^{\prime}$ for the simplicial groupoid $\mathcal{E}$ used above, the argument proceeds as before.

Now suppose that $f$ satisfies (F1) and (F2). We seek to show that $f$ has the right lifting property with respect to the map (A2). In other words, we need to show that the dotted arrow lift exists in the diagram

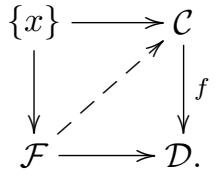

However, the existence of such a lift follows from the fact that the map $f$ satisfies property (F2).

The proof of the following proposition follows just as the analogous statement for simplicial categories [5, 3.1].

Proposition 2.4. The category $\mathcal{S G}$ pd has all small limits and colimits and its class of weak equivalences is closed under retracts and satisfies the "two out of three" property.

Notice that, as for the case of $\mathcal{S C}$, a map $f: \mathcal{C} \rightarrow \mathcal{D}$ satisfies both (F1) and (W1) if and only if it has the right lifting property with respect to the maps in (C1). However, we need the following stronger statement.

Proposition 2.5. A map in $\mathcal{S G}$ pd is a fibration and a weak equivalence if and only if it has the right lifting property with respect to the maps in (C1) and (C2).

Proof. First, we suppose that $f: \mathcal{C} \rightarrow \mathcal{D}$ is a fibration and a weak equivalence. As noted above, it follows from the definitions that $f$ has the right lifting property with respect to the maps in $(\mathrm{C} 1)$. Thus, it remains to show that $f$ has the right lifting property with respect to the map $(\mathrm{C} 2)$, i.e., with respect to the map $\emptyset \rightarrow\{x\}$. However, satisfying such a lifting property is equivalent to being surjective on objects. The fact that $f$ is essentially surjective, or surjective on isomorphism classes of objects, follows from condition (W2). Given this property, the fact that $f$ satisfies condition (F2) guarantees that it is actually surjective. 
Conversely, suppose that $f: \mathcal{C} \rightarrow \mathcal{D}$ has the right lifting property with respect to the maps in $(\mathrm{C} 1)$ and $(\mathrm{C} 2)$. We need to show that $f$ is a fibration and a weak equivalence. As noted above, it follows immediately from the definitions that $f$ satisfies conditions (F1) and (W1).

The fact that $f$ satisfies (W1) implies that the map

$$
\operatorname{Hom}_{\pi_{0} \mathcal{C}}(x, y) \rightarrow \operatorname{Hom}_{\pi_{0} \mathcal{D}}(f x, f y)
$$

is an isomorphism of sets. Furthermore, the fact that $f$ has the right lifting property with respect to the map in $(\mathrm{C} 2)$ guarantees that $f$ is surjective on objects. Therefore, the map $\pi_{0} f: \pi_{0} \mathcal{C} \rightarrow \pi_{0} \mathcal{D}$ is an equivalence of categories; i.e., condition (W2) is satisfied.

It remains to show that $f$ satisfies condition (F2). Since we have already proved that $f$ satisfies (F1) if and only if it has the right lifting property with respect to (A1), and that if $f$ has the right lifting property with respect to (A1) and (A2), then it satisfies (F2), it suffices to show that $f$ has the right lifting property with respect to (A2). However, the map $\{x\} \rightarrow \mathcal{F}$ in (A2) can be written as a composite of a pushout along $\emptyset \rightarrow\{x\}$ followed by pushouts along maps of the form $U_{G} \dot{\Delta}[n] \rightarrow U_{G} \Delta[n]$ for $n=0,1$. But, $f$ has the right lifting property with respect to all such maps since they are in $(\mathrm{C} 1)$ and $(\mathrm{C} 2)$.

Proposition 2.6. A map in $\mathcal{S G}$ pd is an an acyclic cofibration if and only if it has the left lifting property with respect to the fibrations.

The proof of this proposition can be proved formally, just as in the case of simplicial categories [5, 3.3]. It does, however, require the following lemma, whose proof essentially follows the one for $\mathcal{S C}_{\mathcal{O}}$ in $[\mathbf{1 3}, 7.3]$.

Lemma 2.7. The model category $\mathcal{S G} \mathcal{G}_{\mathcal{O}}$ is proper.

Proof. We first prove that $\mathcal{S G} p d_{\mathcal{O}}$ is right proper, namely, that a pullback of a weak equivalence along a fibration is a weak equivalence. However, since fibrations and weak equivalences are defined in terms of fibrations and weak equivalences of mapping spaces, this fact follows from the right properness of $\mathcal{S} \mathcal{S}$ ets $[\mathbf{1 7}, 13.1 .13]$.

To prove that $\mathcal{S G} p d_{\mathcal{O}}$ is left proper, we need to show that the pushout of a weak equivalence along a cofibration is a weak equivalence. Suppose that the following diagram

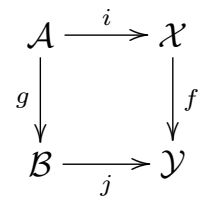

is a pushout diagram with the map $g$ a cofibration and $i$ a weak equivalence. To prove that the map $j$ is a weak equivalence, we can assume that the map $g: \mathcal{A} \rightarrow \mathcal{B}$ is a free map, since cofibrations are retracts of free maps [12, 2.4]. Furthermore, any free map can be written as the colimit of a sequence of free maps for which all of the nondegenerate generators are in the same dimension. So, suppose that $\mathcal{C}$ is an ordinary groupoid, regarded as a simplicial groupoid, and let $\mathcal{C} \otimes \Delta[n]$ denote the 
simplicial groupoid given by the simplicial structure on $\mathcal{S G} p d_{\mathcal{O}}$. Then, it suffices to show that $j$ is a weak equivalence in the diagram (made up of two pushout squares)

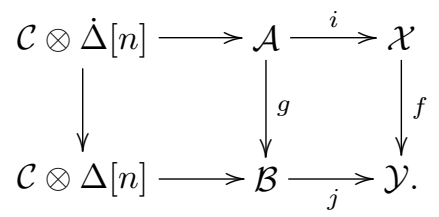

This result follows from technical results on pushouts in $[\mathbf{1 3}, \S 8]$.

Proof of Theorem 2.2. We need to verify the conditions of Theorem 2.1 of the previous paper [4]. The fact that $\mathcal{M}$ has small limits and colimits and that its weak equivalences satisfy the necessary conditions was proved in Proposition 2.4. Condition 1 follows from the same arguments as used for simplicial categories [5, 1.1]. Condition 2 was proved in Proposition 2.3. Condition 3 was proved in Proposition 2.5. Condition 4 was proved in Proposition 2.6.

The following proposition is proved similarly to the analogous result for simplicial categories $[\mathbf{5}, 3.5]$.

Proposition 2.8. The model category $\mathcal{S G}$ pd is right proper.

Proof. Suppose that



is a pullback diagram of simplicial groupoids, where $g: \mathcal{B} \rightarrow \mathcal{D}$ is a fibration and $h: \mathcal{C} \rightarrow \mathcal{D}$ is a Dwyer-Kan equivalence. We would like to show that $f: \mathcal{A} \rightarrow \mathcal{B}$ is a Dwyer-Kan equivalence as well.

We first need to show that $\operatorname{Map}_{\mathcal{A}}(x, y) \rightarrow \operatorname{Map}_{\mathcal{B}}(f x, f y)$ is a weak equivalence of simplicial sets for any objects $x$ and $y$ of $\mathcal{A}$. However, this fact follows since the model category structure on simplicial sets is right proper $[\mathbf{1 7}, 13.1 .4]$.

It remains to prove that $\pi_{0} \mathcal{A} \rightarrow \pi_{0} \mathcal{B}$ is an equivalence of categories. Given what we have proved thus far, it suffices to show that $\mathcal{A} \rightarrow \mathcal{B}$ is essentially surjective on objects.

Consider an object $b$ of $\mathcal{B}$ and its image $g(b)$ in $\mathcal{D}$. Since $\mathcal{C} \rightarrow \mathcal{D}$ is a Dwyer-Kan equivalence, there exists an object $c$ of $\mathcal{C}$ together with an isomorphism $g(b) \cong h(c)$ in $\mathcal{D}$. Since $\mathcal{B} \rightarrow \mathcal{D}$ is a fibration, there exists an object $b^{\prime}$ and isomorphism $b \cong b^{\prime}$ in $\mathcal{B}$ such that $g\left(b^{\prime}\right)=h(c)$. Using the fact that $\mathcal{A}$ is a pullback, we have an isomorphism $b \cong f\left(b^{\prime}, c\right)$, completing the proof.

\section{Invertible Segal spaces}

In this section, we define "groupoid versions" of Rezk's Segal spaces and complete Segal spaces. To do so, we first summarize a few general facts using the category 
$\mathbf{I} \boldsymbol{\Delta}^{o p}$; further details can be found in the previous paper $[\mathbf{4}, \S 4]$. We note that Rezk's original definition of (complete) Segal spaces can be recovered from the definitions in this section by replacing the category $\mathbf{I} \boldsymbol{\Delta}^{o p}$ with the category $\boldsymbol{\Delta}^{o p}$ throughout. The same holds for our definitions of Segal groupoids in the next section. We give fewer details in this section and the following ones, compared to the previous section, due to the fact that most of the proofs are not only similar but actually follow just as in the original cases.

In the category $\mathbf{I} \boldsymbol{\Delta}$, there are maps $\beta^{i}: I[1] \rightarrow I[k]$ given by $0 \mapsto i$ and $1 \mapsto i+1$. We denote the corresponding map in $\mathbf{I} \boldsymbol{\Delta}^{o p}$ by $\beta_{i}: I[k] \rightarrow I[1]$. Each map $\beta_{i}$ induces an inclusion of invertible simplicial sets $I \Delta[1] \rightarrow I \Delta[n]$. We thus define the invertible simplicial space

$$
I G(k)^{t}=\bigcup_{i=0}^{k-1} \beta^{i} I \Delta[1]^{t}
$$

and the inclusion map

$$
\xi^{k}: I G(k)^{t} \rightarrow I \Delta[n]^{t}
$$

Furthermore, this inclusion map induces, for any invertible simplicial space $X$, a map

$$
\operatorname{Map}\left(I \Delta[k]^{t}, X\right) \rightarrow \operatorname{Map}\left(I G(k)^{t}, X\right)
$$

which can be written as the Segal map

$$
\xi_{k}: X_{k} \rightarrow \underbrace{X_{1} \times_{X_{0}} \cdots \times_{X_{0}} X_{1}}_{k} .
$$

(This treatment should be compared to Rezk's in the non-invertible case [27, §4].)

Using $\mathbf{I} \boldsymbol{\Delta}^{o p}$ rather than $\boldsymbol{\Delta}^{o p}$, we still have the injective and projective model structures on the category $\mathcal{S S}$ ets $^{\mathbf{I}}{ }^{o p}$. These model structures can be defined via the inclusion maps $\mathbf{I} \boldsymbol{\Delta}^{o p} \rightarrow \boldsymbol{\Delta}^{o p}$. For the injective structure, then, the generating cofibrations are the maps

$$
\dot{\Delta}[m] \times I \Delta[n]^{t} \cup \Delta[m] \times I \dot{\Delta}[n]^{t} \rightarrow \Delta[m] \times I \Delta[n]^{t}
$$

for all $n, m \geqslant 0$. For the projective structure, the generating cofibrations are the maps

$$
\dot{\Delta}[m] \times I \Delta[n]^{t} \rightarrow \Delta[m] \times I \Delta[n]^{t}
$$

for all $m, n \geqslant 0$.

Definition 3.1. An invertible Segal space is an injective fibrant invertible simplicial space $W$ such that the Segal maps $\xi_{k}$ are weak equivalences of simplicial sets for $k \geqslant 2$.

Define the map

$$
\xi=\coprod_{k \geqslant 1}\left(\xi^{k}: I G(k)^{t} \rightarrow I \Delta[k]^{t}\right) .
$$

Theorem 3.2. Localizing the injective model category structure on the category $\mathcal{S} \mathcal{S e t s}^{\mathbf{I}{ }^{\circ o p}}$ of invertible simplicial spaces, with respect to the map $\xi$, results in a model structure $\mathcal{I} \mathcal{S} S \mathcal{S}_{c}$ in which the fibrant objects are the invertible Segal spaces. 
We can also define a model structure $\mathcal{I} \mathcal{S} e \mathcal{S} p_{f}$ on the category of simplicial spaces by localizing the projective, rather than the injective, model structure with respect to the map $\xi$. In this case the fibrant objects are invertible simplicial spaces fibrant in the projective model structure for which the Segal maps are isomorphisms.

Like a Segal space, an invertible Segal space has a set of "objects," given by the set $W_{0,0}$, and, for any pair $(x, y)$ of objects, a "mapping $\operatorname{space}^{\prime} \operatorname{map}_{W}(x, y)$. This mapping space is defined to be the fiber over $(x, y)$ of the map

$$
W_{1} \stackrel{d_{1} \times d_{0}}{\longrightarrow} W_{0} \times W_{0} .
$$

However, the fact that we are considering an invertible Segal space implies that these mapping spaces have inverses, so that for any $x$ and $y$ objects in $W$, the simplicial sets $\operatorname{map}_{W}(x, y)$ and $\operatorname{map}_{W}(y, x)$ are isomorphic. Thus, all maps are homotopy equivalences and the space $W_{\text {hoequiv }} \subseteq W_{1}$ consisting of "homotopy equivalences" (as defined by Rezk for Segal spaces $[\mathbf{2 7}, \S 5]$ ) is in fact all of $W_{1}$ for an invertible Segal space $W$. (It is helpful here to regard an invertible Segal space simply as a Segal space via the inclusion functor $\boldsymbol{\Delta}^{o p} \rightarrow \mathbf{I} \boldsymbol{\Delta}^{o p}$, in which case Rezk's definitions can be used as is.)

Definition 3.3. An invertible complete Segal space is an invertible Segal space $W$ such that the degeneracy map $s_{0}: W_{0} \rightarrow W_{1}$ is a weak equivalence of simplicial sets.

Notice that in some sense this definition is silly, because an invertible complete Segal space is one for which $W_{0}$ and $W_{1}$ are weakly equivalent, and therefore, using the definition of Segal space, each equivalent to $W_{n}$ for each $n \geqslant 0$. Thus, invertible complete Segal spaces are just, up to weak equivalence, constant simplicial spaces, and equivalent to simplicial sets. We prove this fact more explicitly in Section 5.

However, we can take the same map $\psi: \Delta[0]^{t} \rightarrow E^{t}$ that Rezk used to obtain a complete Segal space model structure on the category of simplicial spaces to obtain an invertible complete Segal space model structure on the category of invertible simplicial spaces. Here, $E$ denotes the nerve of the category $\mathcal{F}$ with two objects and a single isomorphism between these two objects and no other nonidentity morphisms. The map $\psi$ is just the inclusion of one of these objects. The following theorem can then be proved just as Rezk proves the analogous theorem for the complete Segal space model structure $[\mathbf{2 7}, 7.2]$

Theorem 3.4. There is a model structure ICSS on the category of invertible simplicial spaces such that the fibrant objects are the invertible complete Segal spaces.

\section{Segal groupoids}

To define the notion of a Segal groupoid, we again use Segal maps in the context of invertible simplicial spaces. We first used this approach to them in [4], but the idea of a Segal groupoid has also been studied from a slightly different angle by Simpson [28] and Pellissier [25].

Definition 4.1. A Segal pregroupoid is an invertible simplicial space $X$ such that the simplicial set $X_{0}$ is discrete. 
We denote the category of Segal pregroupoids by $\mathcal{S} e \mathcal{G} p d$.

Recall from the previous section that, given an invertible simplicial space $X$, the maps $\beta^{k}$ for $k \geqslant 2$ induce the Segal maps

$$
\xi_{k}: X_{k} \rightarrow \underbrace{X_{1} \times_{X_{0}} \cdots \times_{X_{0}} X_{1}}_{k} .
$$

Definition 4.2. A Segal groupoid is a Segal groupoid $X$ such that the Segal maps $\xi_{k}$ are weak equivalences of simplicial sets for all $k \geqslant 2$.

In the previous paper, we proved that there model category structures on the category of Segal groupoids with a fixed object set $\mathcal{O}$.

Proposition $4.3([4,4.1])$. There is a model category structure $\mathcal{I} S \mathcal{S} p_{\mathcal{O}, f}$ on the category of Segal pregroupoids with a fixed set $\mathcal{O}$ in degree zero in which the weak equivalences and fibrations are given levelwise. Similarly, there is a model category structure $\mathcal{I S S} p_{\mathcal{O}, c}$ on the same underlying category in which the weak equivalences and cofibrations are given levelwise. Furthermore, we can localize each of these model category structures with respect to a map to obtain model structures $\mathcal{I} \mathcal{L S S} p_{\mathcal{O}, f}$ and $\mathcal{I} \mathcal{S} \mathcal{S}_{p_{\mathcal{O}, c}}$ whose fibrant objects are Segal groupoids.

(Recall here that the subscripts $c$ and $f$ are meant to suggest the injective and projective model structures, with the respective letter indicating whether cofibrations or fibrations are given levelwise.)

Furthermore, the following rigidification result holds in this fixed object set case.

Proposition $4.4([4,4.2])$. There is a Quillen equivalence between the model categories $\mathcal{I} \mathcal{L S} \mathcal{S} p_{\mathcal{O}, f}$ and $\mathcal{S G} d_{\mathcal{O}}$.

We conjectured in [4] that this result should still hold when we generalize to the category of all small simplicial groupoids and all Segal groupoids. Before proving this result, we need to establish that we have the necessary model structures for Segal groupoids, i.e., model structures on the category of Segal pregroupoids in which the fibrant objects are Segal groupoids.

The first step in finding such model structures is modifying the generating cofibrations and generating acyclic cofibrations of $\mathcal{S} \mathcal{S} e t s_{c}^{\mathbf{I} \boldsymbol{\Delta}^{o p}}$ and $\mathcal{S} \mathcal{S} e t s_{f}^{\mathbf{I} \boldsymbol{\Delta}^{o p}}$ so that they are maps between Segal pregroupoids rather than maps between arbitrary invertible simplicial spaces.

Recall that for $\mathcal{S} \mathcal{S e t} s_{c}^{\mathbf{I}{ }^{o p}}$, a set of generating cofibrations is given by

$$
\left\{\dot{\Delta}[n] \times I \Delta[n]^{t} \cup \Delta[m] \times I \dot{\Delta}[n]^{t} \rightarrow \Delta[m] \times I \Delta[n]^{t} \mid m, n \geqslant 0\right\} .
$$

To find the set that we need, we apply a reduction functor $(-)_{r}$ which makes the space in degree 0 discrete and then check potentially problematic values of $n$ and $m$, as in $[\mathbf{7}, \S 4]$. Thus, we obtain the set $I_{c}$ given by

$$
\begin{aligned}
\left\{\left(\dot{\Delta}[m] \times I \Delta[n]^{t} \cup \Delta[m]\right.\right. & \left.\times I \dot{\Delta}[n]^{t}\right)_{r} \\
& \left.\rightarrow\left(\Delta[m] \times I \Delta[n]^{t}\right)_{r} \mid m \geqslant 0 \text { when } n \geqslant 1, n=m=0\right\}
\end{aligned}
$$

as a potential set of generating cofibrations. 
For the modification of the generating cofibrations of $\mathcal{S} \mathcal{S} e t s_{f}^{\mathbf{I} \boldsymbol{\Delta}^{o p}}$, we need to take a different approach. To do so, we make the following definitions. Let $I P_{m, n}$ be the pushout in the diagram

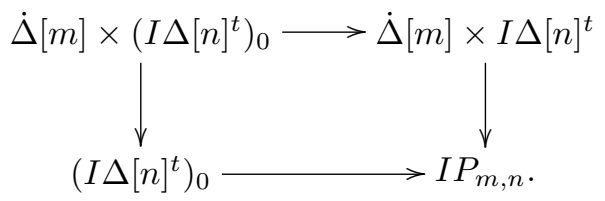

If we replace $\dot{\Delta}[m]$ with $\Delta[m]$ in the above diagram, we denote the pushout $I Q_{m, n}$. We then define a set of generating cofibrations for $\mathcal{S} e \mathcal{G} p d_{f}$ to be

$$
I_{f}=\left\{I_{m, n}: I P_{m, n} \rightarrow I Q_{m, n} \mid m, n \geqslant 0\right\} .
$$

Now, to give the definitions of the desired weak equivalences for our model structures, we establish an appropriate "localization" functor. Since our model structures are not actually obtained by localizing another model structure, this functor is not technically a localization, but it is analogous to the fibrant replacement functor in $\mathcal{I S e} \mathcal{S} p_{f}$, which is obtained from localization, and it does turn out to be a fibrant replacement functor in $\mathcal{S} e \mathcal{G} p d_{f}$.

For the invertible Segal space model structure $\mathcal{I} \mathcal{S} S p_{c}$, a choice of generating acyclic cofibrations is the set

$$
\left\{V[m, k] \times I \Delta[n]^{t} \cup \Delta[m] \times I G(n)^{t} \rightarrow \Delta[m] \times I \Delta[n]^{t} \mid n \geqslant 0, m \geqslant 1,0 \leqslant k \leqslant m\right\} .
$$

To have these maps defined between Segal pregroupoids rather than between arbitrary invertible simplicial spaces, we restrict to the case where $n \geqslant 1$. As in [7, §5] it can be shown that taking an colimit of iterated pushouts along all such maps results in a Segal groupoid which is also an invertible Segal space. We will denote this functor $L_{c}$. There is an analogous functor in the model category $\mathcal{I} \mathcal{S} S p_{f}$ which we denote $L_{f}$.

Thus, using the fact that an invertible Segal space $X$ has "objects," "mapping spaces," and a "homotopy category" $\operatorname{Ho}(X)$ (again, as given by Rezk in [27, §5]), we can define the classes of maps we need for the model structure $\mathcal{S} \mathcal{G} p d_{c}$. First, we define a Dwyer-Kan equivalence of invertible Segal spaces to be a map $f: W \rightarrow Z$ such that

- the $\operatorname{map}_{\operatorname{map}_{W}}(x, y) \rightarrow \operatorname{map}_{Z}(f x, f y)$ is a weak equivalence of simplicial sets for any pair of objects $x$ and $y$ of $W$, and

- the map $\mathrm{Ho}(W) \rightarrow \mathrm{Ho}(Z)$ is an equivalence of categories.

Theorem 4.5. There is a model category structure $\mathcal{S} e \mathcal{G} p d_{c}$ on the category of Segal pregroupoids such that

1. a weak equivalence is a map $f: X \rightarrow Y$ such that the induced map $L_{c} X \rightarrow L_{c} Y$ is a Dwyer-Kan equivalence of Segal spaces,

2. a cofibration is a monomorphism (so every Segal pregroupoid is cofibrant), and

3. a fibration is a map with the right lifting property with respect to the maps which are cofibrations and weak equivalences. 
This theorem can be proved using as generating cofibrations the set $I_{c}$ defined above and as generating acyclic cofibrations the set $J_{c}=\{i: A \rightarrow B\}$ of representatives of isomorphism classes of maps in $\mathcal{S} e \mathcal{G} p d$ satisfying

1. for all $n \geqslant 0$, the spaces $A_{n}$ and $B_{n}$ have countably many simplices, and

2. the map $i: A \rightarrow B$ is a monomorphism and a weak equivalence.

Given these definitions, the proof of the existence of the model structure $\mathcal{S} e \mathcal{G} p d_{c}$, while technical, follows just as the proof for the model structure $\mathcal{S e C} a t_{c}[\mathbf{7}, 5.1]$.

The other model structure, $\mathcal{S} e \mathcal{G} p d_{f}$, has the same weak equivalences as $\mathcal{S} e \mathcal{G} p d_{c}$, but not all monomorphisms are cofibrations. Instead, we take the maps of Segal pregroupoids which are cofibrations in the projective model structure $\mathcal{S} \mathcal{S}_{e t} s_{f}^{\mathbf{I} \Delta^{o p}}$.

Theorem 4.6. There is a cofibrantly generated model structure $\mathcal{S} e \mathcal{G} p d_{f}$ on the category of Segal pregroupoids given by the following classes of morphisms:

1. the weak equivalences are the same as those of $\mathcal{S} \mathcal{G}$ pd $d_{c}$,

2. the cofibrations are the maps which can be obtained by taking iterated pushouts along the maps in the set $I_{f}$, and

3. the fibrations are the maps with the right lifting property with respect to the maps which are both cofibrations and weak equivalences.

Here, the set of generating cofibrations is $I_{f}$, and the set of generating acyclic cofibrations is given by the set $J_{f}$ which is defined analogously to $J_{c}$ but using the new definition of cofibration. Again, the proof follows just as in $[\mathbf{7}, 7.1]$.

\section{Quillen equivalences}

Here, we show that we still have Quillen equivalences between these various model categories. In fact, the proofs that we give in [7] continue to hold. Here we give a sketch of what the various functors are connecting these categories. We begin with the simplest example. Throughout, the topmost arrow indicates the left Quillen functor.

Proposition 5.1. The identity functor

$$
i d: \mathcal{S} e \mathcal{G} p d_{f} \rightleftarrows \mathcal{S} e \mathcal{G} p d_{c}: i d
$$

is a Quillen equivalence of model categories.

To compare the model categories $\mathcal{S} e \mathcal{G} p d_{c}$ and $\mathcal{I C S S}$, first notice that we can take the inclusion functor

$$
I: \mathcal{S e} \mathcal{G} p d_{c} \rightarrow \mathcal{I C S S} \text {. }
$$

This functor has a right adjoint $R$ given as follows.

Recall that, given any simplicial space $X$, we can consider its 0-coskeleton, denoted $\operatorname{cosk}_{0}(X)[\mathbf{2 6}, \S 1]$. Let $W$ be an invertible simplicial space, and regard $W_{0}$ and $W_{0,0}$ as constant simplicial spaces. Consider the invertible simplicial spaces $U=\operatorname{cosk}_{0}\left(W_{0}\right)$ 
and $V=\operatorname{cosk}_{0}\left(W_{0,0}\right)$ and the natural maps $W \rightarrow U \leftarrow V$. Define $R W$ to be the pullback of the diagram

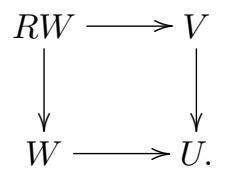

As in $[7, \S 6]$, we can see that $R$ is in fact a right adjoint and that the following result holds:

Proposition 5.2. The adjoint pair

$$
I: \mathcal{S} e \mathcal{G} p d_{c} \rightleftarrows \mathcal{I C S S}: R
$$

is a Quillen equivalence of model categories.

To prove the existence of a Quillen equivalence between $\mathcal{S G} p d$ and $\mathcal{S} e \mathcal{G} p d_{f}$, we first notice that via the nerve functor a simplicial groupoid can be regarded as a strictly local object in the category of $\mathbf{I} \boldsymbol{\Delta}^{o p}$ diagrams of simplicial sets with respect to the map $\xi$ used to define the invertible Segal space model structure. Thus, the nerve functor $R: \mathcal{S G} p d \rightarrow \mathcal{S} e \mathcal{G} p d_{f}$ can be shown to have a left adjoint via the following lemma.

Lemma $5.3([6,5.6])$. Consider two categories, the category of all diagrams $X: \mathcal{D} \rightarrow$ $\mathcal{S S e t s}$ and the category of strictly local diagrams with respect to some set of maps $S=\{f: A \rightarrow B\}$. The forgetful functor from the category of strictly local diagrams to the category of all diagrams has a left adjoint.

Denoting this left adjoint $F$, the following result once again follows from the same arguments given in $[\mathbf{7}, 8.6]$.

Theorem 5.4. The adjoint pair

$$
F: \mathcal{S} e \mathcal{G} p d_{f} \rightleftarrows \mathcal{S G} p d: R
$$

is a Quillen equivalence of model categories.

However, in this case we also have the following additional result, emphasizing the idea that an $(\infty, 0)$-category should just be a space (or simplicial set).

Theorem 5.5. Let $T: \mathcal{I C S S} \rightarrow \mathcal{S S}$ ets be the functor taking an invertible complete Segal space $W$ to its 0 -space $W_{0}$. This functor has a left adjoint given by the functor $C: \mathcal{S S e t s} \rightarrow \mathcal{I C S S}$ taking a simplicial set $K$ to the constant invertible simplicial space $C K$. This adjoint pair gives a Quillen equivalence of model categories.

Proof. It is not hard to show that $C$ is left adjoint to $T$. To prove that this adjoint pair is a Quillen pair, we observe that the left adjoint functor $C$ preserves cofibrations because they are just monomorphisms in each category. It also preserves acyclic cofibrations because the image of a weak equivalence of simplicial sets is a Reedy weak equivalence of simplicial spaces, and therefore also a weak equivalence in $\mathcal{I C S S}$.

By the same reasoning, $C$ also reflects weak equivalences between cofibrant objects. Thus, to prove that we have a Quillen equivalence it remains to show that the map 
$C(T W) \rightarrow W$ is a weak equivalence for any invertible complete Segal space $W$. However, $C(T W)=C\left(W_{0}\right)$, the constant invertible simplicial set which has $W_{0}$ in each degree. However, if $W$ is an invertible complete Segal space, then the spaces in each degree are all weakly equivalent. Thus, the desired map is a weak equivalence.

Again, we note that this result is not surprising, in that Dwyer and Kan proved that $\mathcal{S G}$ d is Quillen equivalent to $\mathcal{S} \mathcal{S}$ ets in [12], but this particular Quillen equivalence with $\mathcal{I C S S}$ makes the relationship especially clear.

We conclude this section by noting some relationships between models for invertible homotopy theories as given in this paper and models for homotopy theories. Recall that the inclusion map $\boldsymbol{\Delta}^{o p} \rightarrow \mathbf{I} \boldsymbol{\Delta}^{o p}$ induces a map

$$
\mathcal{S} \operatorname{Sets}^{\mathbf{I} \Delta^{o p}} \rightarrow \mathcal{S S}_{\text {ets }^{\Delta^{o p}}}
$$

which has an "invert" map as a left adjoint. This adjoint pair is in fact a Quillen pair when we consider the respective injective model category structures on the two categories. Furthermore, we still have a Quillen pair

$$
\mathcal{I C S S} \leftrightarrows \mathcal{C S S}
$$

between the localized model categories.

This adjoint relationship between $\mathcal{C S S}$ and $\mathcal{I C S S}$ (where the "invert" map is the left adjoint) can be contrasted with an Quillen pair between $\mathcal{C S S}$ and $\mathcal{S} \mathcal{S}$ ets for which the analogous map is the right adjoint. The map $\mathcal{C S S} \rightarrow \mathcal{S S}$ ets given by taking a simplicial space $X$ to the simplicial set $X_{0}$ can be shown to have a left adjoint (giving, essentially, a constant simplicial space). It can be shown to be a right Quillen functor because if $X$ is a fibrant object (i.e., a complete Segal space and therefore Reedy fibrant), then $X_{0}$ is fibrant in $\mathcal{S} \mathcal{S}$ ets as well, and because weak equivalences between complete Segal spaces give weak equivalences between 0 -spaces.

\section{A Bousfield approach to Segal groupoids and invertible complete Segal spaces}

As in [4], we can also use a different approach to defining Segal groupoids and invertible (complete) Segal spaces. The idea behind this method is, when moving from the ordinary Segal case to the invertible one, to change the projections used to define the Segal maps, an idea used by Bousfield [9].

In the category $\boldsymbol{\Delta}$, consider the maps $\gamma^{k}:[1] \rightarrow[n]$ given by $0 \mapsto 0$ and $1 \mapsto k+1$ for any $0 \leqslant k<n$. Just as the maps $\alpha^{k}$ are used to define the ordinary Segal maps $\varphi_{k}$, we can use the maps $\gamma^{k}$ to define the Bousfield-Segal maps

$$
\chi_{n}: X_{n} \rightarrow \underbrace{X_{1} \times_{X_{0}} \cdots \times_{X_{0}} X_{1}}_{n}
$$

for each $n \geqslant 2$.

Definition 6.1. A Bousfield-Segal space is a Reedy fibrant simplicial space $W$ satisfying the condition that the Bousfield-Segal maps $\chi_{n}$ are weak equivalences for $n \geqslant 2$. 
Notice that Bousfield-Segal spaces are really just another way to think about invertible Segal spaces. We have given them a different name here to distinguish them from our previous definition; when we consider model category structures the distinction is more important, since the underlying category here is that of simplicial spaces, rather than that of invertible simplicial spaces.

To define a model category structure on the category of simplicial spaces in which the fibrant objects are Bousfield-Segal spaces, we need to define an appropriate map with which to localize the Reedy model structure. For $\gamma^{i}$ defined as above, define

$$
H(k)^{t}=\bigcup_{i=1}^{k-1} \gamma^{i} \Delta[1]^{t}
$$

and the inclusion map

$$
\chi^{k}: H(k)^{t} \rightarrow \Delta[k]^{t} .
$$

Combining these maps for all values of $k$, we obtain a map

$$
\chi=\coprod_{k \geqslant 1}\left(\chi^{k}: H(k)^{t} \rightarrow \Delta[k]^{t}\right) .
$$

Theorem 6.2. Localizing the Reedy model structure with respect to the map $\chi$ results in a model structure $\mathcal{B S e S} p_{c}$ in which the fibrant objects are the Bousfield-Segal spaces.

As usual, we can also localize the projective model structure with respect to the map $\chi$ to obtain a model category $\mathcal{B S} e \mathcal{S} p_{f}$.

The properties of invertible Segal spaces discussed previously continue to hold for Bousfield-Segal spaces. In particular, we have the following definition.

Definition 6.3. A complete Bousfield-Segal space is a Bousfield-Segal space $W$ for which the degeneracy map $s_{0}: W_{0} \rightarrow W_{1}$ is a weak equivalence.

Recall the map $\psi: \Delta[0]^{t} \rightarrow E^{t}$ used to define the (invertible) complete Segal space model structure. We use it again here to establish what we call the complete BousfieldSegal space model structure on the category of simplicial spaces.

Theorem 6.4. Localizing the model structure $\mathcal{B S} S \mathcal{S} p_{c}$ with respect to the map $\psi$ results in a model structure $\mathcal{C B S S}$ in which the fibrant objects are the complete Bousfield-Segal spaces.

We now turn to the Bousfield approach to Segal groupoids. We gave the following definition in $[4, \S 6]$.

Definition 6.5. A Bousfield-Segal category is a Segal precategory for which the Bousfield-Segal maps $\chi_{n}$ are weak equivalences for each $n \geqslant 2$.

In [4], we consider the fixed object set case, in which we look at Bousfield-Segal categories with a given set $\mathcal{O}$ in degree zero. In particular, we defined a fixed-object version of the map $\chi$ as follows:

$$
\chi_{\mathcal{O}}=\coprod_{k \geqslant 0}\left(\chi_{\mathcal{O}}^{k}: H(k)_{\mathcal{O}}^{t} \rightarrow \Delta[k]_{\mathcal{O}}^{t} \cdot\right)
$$


Proposition 6.6 ([4, 6.1]). Localizing the category $\mathcal{S} \mathcal{S}_{\mathcal{O}, f}$ with respect to the map $\chi_{\mathcal{O}}$ results in a model structure $\mathcal{L}_{B} \mathcal{S} \mathcal{S} p_{\mathcal{O}, f}$ whose fibrant objects are Bousfield-Segal categories. Similarly, localizing $\mathcal{S} \mathcal{S} p_{\mathcal{O}, c}$ with respect to $\chi_{\mathcal{O}}$ results in a model category we denote $\mathcal{L}_{B} \mathcal{S} S p_{\mathcal{O}, c}$.

Using these model structures, we can proceed to the more general case, in which we have model structures $\mathcal{B S e C} a t_{c}$ and $\mathcal{B S e C} a t_{f}$ on the category of Segal precategories in which the fibrant objects are Bousfield-Segal categories. We first consider $\mathcal{B S e C} a t_{c}$, which is analogous to $\mathcal{S e C} a t_{c}$.

Considering the acyclic cofibrations given by the set

$$
\left\{V[m, k] \times \Delta[n]^{t} \cup \Delta[m] \times H(n)^{t} \rightarrow \Delta[m] \times \Delta[n]^{t}\right\}
$$

for $n \geqslant 1, m \geqslant 1$, and $0 \leqslant k \leqslant m$ enables us to define a "localization" functor $L_{B, c}$ taking a Segal precategory to a Bousfield-Segal category by taking a colimit of pushouts along the maps of this set.

Theorem 6.7. There is a model category structure $\mathcal{B S e C}$ at $t_{c}$ on the category of Segal precategories such that

1. a weak equivalence is a map $f: X \rightarrow Y$ such that the induced map $L_{B, c} X \rightarrow$ $L_{B, c} Y$ is a Dwyer-Kan equivalence of Bousfield-Segal spaces,

2. a cofibration is a monomorphism, and

3. a fibration is a map which has the right lifting property with respect to the maps which are cofibrations and weak equivalences.

In particular, in this model structure, the cofibrations should be monomorphisms so that all objects are cofibrant. Therefore, we can use the same set of generating cofibrations,

$$
I_{c}=\left\{\left(\dot{\Delta}[m] \times \Delta[n]^{t} \cup \Delta[m] \times \dot{\Delta}[n]^{t}\right)_{r} \rightarrow\left(\Delta[m] \times \Delta[n]^{t}\right)_{r}\right\},
$$

where $m \geqslant 0$ when $n \geqslant 1$, and when $n=m=0$, that we used for $\mathcal{S e C}_{a} t_{c}$ in $[\mathbf{7}, \S 5]$. We can define a set of generating acyclic cofibrations very similarly, namely, by a set of representatives of isomorphism classes of maps $i: A \rightarrow B$ which are cofibrations and weak equivalences such that for all $n \geqslant 0$ the simplicial sets $A_{n}$ and $B_{n}$ have only countably many simplices. Notice that this definition only differs from the one in $[\mathbf{7}]$ in that we weak equivalences are defined here in terms of the functor $L_{B, c}$ rather than by the functor $L_{c}$ taking a Segal precategory to a Segal category.

Given these generating sets, the proof of the existence of this model structure follows just as in $[\mathbf{7}, 5.1]$. Once again, we also have a companion model structure $\mathrm{BSeC}_{f}$.

For this model structure, we use a functor $L_{B, f}$ taking a Segal precategory to a Bousfield-Segal category which is fibrant in the projective, rather than the Reedy model structure, and then define the weak equivalences in terms of this functor. However, as before, it turns out that the two functors define the same class of weak equivalences $[\mathbf{7}, \S 7]$. Just as in $\mathcal{S e C} a t_{f}$, we make use of the set

$$
I_{f}=\left\{P_{m, n} \rightarrow Q_{m, n} \mid m, n \geqslant 0\right\} .
$$


Theorem 6.8. There is a model category structure $\mathcal{B S e C} \operatorname{Ct}_{f}$ on the category of Segal precategories such that

1. weak equivalences are the same as those in $\mathcal{B S e C a t}_{c}$,

2. a cofibration is a map which can be obtained by taking iterated pushouts along the maps in the set $I_{f}$, and

3. a fibration is a map which has the right lifting property with respect to the maps which are cofibrations and weak equivalences.

Now, we have the following results, analogous to those of the previous section.

Proposition 6.9. The identity functor induces a Quillen equivalence

$$
i d: \mathcal{B S e C} a t_{f} \rightleftarrows \mathcal{B S e C} a t_{c}: i d \text {. }
$$

Theorem 6.10. The inclusion functor

$$
I: \mathrm{BSeC}_{\mathrm{c}} \rightarrow \mathcal{C B S S}
$$

has a right adjoint, and this adjoint pair is a Quillen equivalence.

As described in the previous section, the right adjoint here, applied to an object $W$ of $\mathcal{C B S S}$, is given by a pullback of the diagram

$$
W \rightarrow \operatorname{cosk}_{0}\left(W_{0}\right) \leftarrow \operatorname{cosk}_{0}\left(W_{0,0}\right) .
$$

The proof that this map is adjoint to the inclusion map and that the adjoint pair is in fact a Quillen equivalence of model categories follows just as the one given in [7, $\S 6]$.

We can again make use of Lemma 5.3 and an argument like the one in $[\mathbf{7}, 8.6]$ to prove the following result.

Theorem 6.11. The nerve functor

$$
R: \mathcal{S G} p d \rightarrow \mathcal{B S e C} a t_{f}
$$

has a left adjoint, and this adjoint pair is a Quillen equivalence.

Lastly, we can compare the model structure $\mathcal{C B S} S$ to the model structure on simplicial sets just as we did in the proof of Theorem 5.5.

Theorem 6.12. Let $T: \mathcal{C B S S} \rightarrow \mathcal{S S}$ ets be the functor taking an complete BousfieldSegal space $W$ to its 0 -space $W_{0}$. This functor has a left adjoint $C: \mathcal{S S}$ ets $\rightarrow \mathcal{C B S S}$ taking a simplicial set $K$ to the constant invertible simplicial space $C K$. This adjoint pair gives a Quillen equivalence of model categories.

\section{References}

[1] J. Baez, The homotopy hypothesis, talk given at the Fields Institute, January 2007, slides available at http://www.math.ucr.edu/home/baez/homotopy/.

[2] C. Barwick, private communication.

[3] C. Berger, A cellular nerve for higher categories, Adv. Math. 169 (2002), no. 1, $118-175$. 
[4] J.E. Bergner, Adding inverses to diagrams encoding algebraic structures, Homology, Homotopy and Applications 10 (2008), no. 2, 149-174.

[5] J.E. Bergner, A model category structure on the category of simplicial categories, Trans. Amer. Math. Soc. 359 (2007), no. 5, 2043-2058.

[6] J.E. Bergner, Rigidification of algebras over multi-sorted theories, Algebr. Geom. Topol. 6 (2006) 1925-1955.

[7] J.E. Bergner, Three models for the homotopy theory of homotopy theories, Topology 46 (2007), no. 4, 397-436.

[8] G. Biedermann, On the homotopy theory of $n$-types, preprint Homology, Homotopy and Applications 10 (2008), no. 1, 305-325.

[9] A.K. Bousfield, The simplicial homotopy theory of iterated loop spaces, unpublished manuscript.

[10] D.-C. Cisinski, Batanin higher groupoids and homotopy types, in Categories in algebra, geometry and mathematical physics, 171-186, Contemp. Math. 431, American Mathematical Society, Providence, RI, 2007.

[11] W.G. Dwyer and D.M. Kan, Function complexes in homotopical algebra, Topology 19 (1980), 427-440.

[12] W.G. Dwyer and D.M. Kan, Homotopy theory and simplicial groupoids, Proc. Kon. Acad. van Wetensch. A87 (1984) no. 4, 379-385.

[13] W.G. Dwyer and D.M. Kan, Simplicial localizations of categories, J. Pure Appl. Algebra 17 (1980), no. 3, 267-284.

[14] W.G. Dwyer and J. Spalinski, Homotopy theories and model categories, in Handbook of Algebraic Topology, 73-126, North-Holland, Amsterdam, 1995. 1995.

[15] P.G. Goerss and J.F. Jardine, Simplicial Homotopy Theory, Progress in Mathematics 174, Birkhäuser Verlag, Basel, 1999.

[16] A. Grothendieck, Pursuing stacks, letter to D. Quillen, available at www.grothendieckcircle.org.

[17] P.S. Hirschhorn, Model categories and their localizations, Mathematical Surveys and Monographs 99, American Mathematical Society, Providence, RI, 2003.

[18] M. Hovey, Model categories, Mathematical Surveys and Monographs 63, American Mathematical Society, Providence, RI, 1999.

[19] A. Joyal, Simplicial categories vs quasi-categories, in preparation.

[20] A. Joyal, The theory of quasi-categories I, in preparation.

[21] A. Joyal and M. Tierney, Quasi-categories vs Segal spaces, in Categories in algebra, geometry and mathematical physics, Contemp. Math. 431 (2007), 277326 .

[22] J. Lurie, Higher topos theory, preprint available at math. CT/0608040.

[23] S. Mac Lane, Categories for the working mathematician, Second edition, Graduate Texts in Mathematics 5, Springer-Verlag, New York, 1998.

[24] S. Paoli, Semistrict Tamsamani $n$-groupoids and connected $n$-types, preprint available at math.AT/0701655. 
[25] R. Pellissier, Catégories enrichies faibles, preprint available at math.AT/ 0308246.

[26] C.L. Reedy, Homotopy theory of model categories, unpublished manuscript, available at http://www-math.mit.edu/ ${ }^{\sim}$ psh.

[27] C. Rezk, A model for the homotopy theory of homotopy theory, Trans. Amer. Math. Soc. 353 (2001), no. 3, 973-1007.

[28] C. Simpson, Effective generalized Seifert-Van Kampen: how to calculate $\Omega X$, preprint available at q-alg/9710011.

[29] Z. Tamsamani, Equivalence de la théorie homotopique des $n$-groupoïdes et celles des espaces topologiques $n$-tronqués, preprint available at alg-geom/ 9607010.

Julia E. Bergner bergnerj@member .ams.org

Department of Mathematics, University of California, Riverside, CA 92521, USA

Previous address: Department of Mathematics, Kansas State University, 138 Cardwell Hall, Manhattan, KS 66506, USA 